\title{
Study of Elastic Moduli of Semiconductors with Defects by the Statistical Moment Method*
}

\author{
$\mathrm{Vu}$ Van Hung ${ }^{\dagger}$ \\ Faculty of Phyics, Hanoi University of Education I, 136 Xuan Thuy, Cau Giay, Hanoi, Vietnam \\ Le Dai Thanh and Ngo Thu Huong \\ Faculty of Physics, Hanoi University of Science, VNU, \\ 334 Nguyen Trai, Thanh Xuan, Hanoi, Vietnam
}

(Received 7 December 2009; Accepted 3 May 2010; Published 27 December 2011)

\begin{abstract}
The elastic moduli of semiconductors at finite temperatures have been studied using the statistical moment method. The Young, bulk and shear moduli of the semiconductor with point defects like Si crystal are calculated as a function of the temperature. We discuss the temperature dependence of the elastic moduli of Si crystal with defects and compare the calculated elastic moduli with the experimental results.

[DOI: 10.1380/ejssnt.2011.499]
\end{abstract}

Keywords: Elastic moduli; Defect; Semiconductor

\section{INTRODUCTION}

The point defects in crystals including the vacancies play an important role in many properties of material. Thus an investigation of the point defects in solid and in influence of the vacancies on the mechanical properties of material are of special interest [1-3]. It is the purpose of the present article to study the elastic moduli of semiconductors with point defects using the moment method in the quantum statistical mechanics, hereafter referred to as the statistical moment method (SMM) [3-8]. So far a number of theoretical approaches have been proposed for the studies of dynamical elastic properties of metals and alloys.

In this paper, we present a new theoretical scheme on the elastical moduli of semiconductors with defects including the temperature effects based on the SMM. With the use of the SMM, the thermodynamic quantities such as the equilibrium concentration $n_{V}$ of vacancies and elastic moduli can be derived using the analytic expressions of the Helmholtz free energy of the given system. When analyzing the mechanical properties of semiconductors, especially those of the high- temperature semiconductors, it is essential to take into account the temperature effects since they depend strongly and sensitively on temperature.

The present paper is organized as follows: in Section II, we present the principles of calculations for the elastic moduli of semiconductors with point defects at finite temperatures, including the anharmonicity of thermal lattice vibrations. The results of numerical calculations and related discussions are given in Section III.

\footnotetext{
* This paper was presented at the International Workshop on Advanced Materials and Nanotechnology 2009 (IWAMN2009), Hanoi University of Science, VNU, Hanoi, Vietnam, 24-25 November, 2009. †Corresponding author: huongnt_kvl@vnu.edu.vn
}

\section{PRINCIPLES OF CALCULATIONS}

A. Free energy and equilibrium concentration of non interaction vacancies of semiconductors

The Gibbs free energy of a mono atomic crystals consisting of $N$ atoms and $n \ll N$ vacancies has the form:

$$
G(T, P)=G_{0}+n g_{V}^{f}-T S_{C}^{n},
$$

Where $G_{0}(T, P)$ is the Gibbs free energy of the perfect crystals containing $N$ atoms, $g_{V}^{f}(T, P)$ is the Gibbs energy change on forming a single vacancy, $S_{C}^{n}-$ the entropy of mixing:

$$
S_{C}^{n}=k_{B} \ln \frac{(N+n) !}{N ! n !}
$$

$T$ and $k_{B}$ are the temperature and Boltzmann constant, respectively. From eq.(1) we obtain an expression of the Helmholtz freee energy of the crystals at the pressure $P=$ 0 :

$$
\psi=\psi_{0}+n g_{V}^{f}-T S_{C}^{n}
$$

$\psi_{0}$ is the free energy of the perfect crystals and given from the SMM [4] as

$$
\begin{gathered}
\psi_{0}=N\left\{U_{0}+3 \theta\left[x+\ln \left(1-e^{-2 x}\right)\right]\right\} \\
U_{0}=\frac{1}{2} \sum_{i} \phi_{i 0}\left(\left|a_{i}\right|\right) \\
x=\frac{\hbar \omega}{2 \theta}, \theta=k_{B} T
\end{gathered}
$$

where $a_{i}$ is the equilibrium position of the $i^{t h}$ particle and $\phi_{i 0}$ is the interaction potential energy between $z e r o^{t h}$ 
and $i^{\text {th }}$ particles. Minimizing $G$, eq.(1) with respect to $n_{V}=\frac{n}{N}$, that means $\left(\frac{\partial G}{\partial n_{V}}\right)_{T, P, N}=0$ leads to the equilibrium concentration $n_{V}$ of non interaction vacancies:

$$
n_{V}=\exp \left\{-\frac{g_{V}^{f}}{\theta}\right\}
$$

where the Gibbs energy change $g_{V}^{f}$ is given as

$$
g_{V}^{f}=-U_{0}+\Delta \psi_{0}^{*}+P \Delta V,
$$

To calculate the interaction energy of the perfect crystal $U_{0}$, we use the many body potentials and take into account the contributions up to the second nearest neighbors. In eq. (6), $\Delta \psi_{0}^{*}$ denotes the change in the Helmholtz freee energy of the central atom which creates a vacancy by moving itself to the certain sinks (e.g., crystal surface, or to the core region of the dislocation and grain boundary) in the crystal

$$
\Delta \psi_{0}^{*}=\psi_{0}^{*^{\prime}}-\psi_{0}^{*} \equiv(B-1) \psi_{0}^{*},
$$

where $\psi_{0}^{*^{\prime}}$ denotes the free energy of the central atom after moving to a certain sink sites in the crystal. In this respect, it is noted that the vacancy formation energies of the real crystals are measured experimentally as an average value over all those values corresponding to the possible sink sites.

Note that the parameter $B>1$ because the Helmholtz free energy of an atom of crystal $\psi_{0}^{*}<0$ and we can choose the free energy change of an particle when leaving from the node of a lattice on forming a single vacancy $\Delta \psi_{0}^{*}<0$.

In the case of the pressure $P=0$, since the Gibbs energy change $g_{V}^{f}>0$, from (6) and (7) we obtain

$$
B<1+\frac{U_{0}}{\psi_{0}^{*}}
$$

Therefore, we can find the parameter $B$ from (8) and the condition $B>1$. In the present study, we take the average value for $B$ as

$$
B \approx 1+\frac{1}{2} \frac{U_{0}}{\psi_{0}^{*}}
$$

From (6), (7), (9) and (3), it is easy to obtain the Gibbs energy change $g_{V}^{f}(T, 0)$ and the Helmholtz free energy at the pressure $P=0$ :

$$
\begin{gathered}
g_{V}^{f}(T, 0) \approx-\frac{U_{0}}{2} ; \\
\psi \approx \psi_{0}-N n_{V} \frac{U_{0}}{2}-T S_{C}^{n} .
\end{gathered}
$$

\section{B. Elastic moduli of semiconductors with defects}

In this subsection, we outline the calculation of the elastic moduli of crystals with defects at finite temperatures with the use of the SMM. For a uniformly deformed crystal, in which strains are infinitesimal so that Hooke's Law is obeyed, the work $W$ done per unit volume in deforming the crystal (elastic strain energy density) $E_{\text {elas }}=W / V$ of the crystal can be calculated by evaluating the changes in the Helmholtz free energy $\Delta \psi$ due to the uniform elastic deformations [8].

The relation of the Helmholtz free energies and the Young modulus $E$ can be written as [9]:

$$
\psi^{P}=\psi+\frac{E \varepsilon^{2}}{2},
$$

where $\psi^{P}$ denotes the Helmholtz free energy for unit volume in deforming and $\varepsilon$ is the elastic strain.

The temperature dependence of Young modulus of crystals with defects are calculated using the expression of the Helmholtz free energy $\psi$ of eq.(11). The second derivative of the Helmholtz free energy $\psi^{P}(12)$ with respect to the strain $\varepsilon$ is calculated as:

$$
\begin{aligned}
E= & \frac{\partial^{2} \psi^{P}}{\partial \varepsilon^{2}} \\
= & E_{0}-\frac{N}{2 V} n_{V}\left\{\frac{1}{\theta}\left(\frac{\partial U_{0}}{\partial \varepsilon}\right)^{2}+\frac{\partial^{2} U_{0}}{\partial \varepsilon^{2}}\right. \\
& \left.+\frac{U_{0}}{2 \theta}\left[\frac{1}{\theta}\left(\frac{\partial U_{0}}{\partial \varepsilon}\right)^{2}+\frac{\partial^{2} U_{0}}{\partial \varepsilon^{2}}\right]\right\}
\end{aligned}
$$

where $E_{0}$ denotes the Young modulus of perfect crystal:

$$
E_{0}=\frac{1}{V} \frac{\partial^{2} \psi_{0}}{\partial \varepsilon^{2}}
$$

The derivatives $\frac{\partial U_{0}}{\partial \varepsilon}$ and $\frac{\partial^{2} U_{0}}{\partial \varepsilon^{2}}$ appearing in eq.(13) are calculated by using the relation [9]

$$
\frac{\partial a}{\partial \varepsilon}=2 a_{0}(1+\varepsilon) ; \frac{\partial^{2} a}{\partial \varepsilon^{2}}=2 a_{0},
$$

where $a_{0}$ and $a$ are the nearest neighbor distances of the system in the case without and with external forces $P$ at zero temperature, respectively. Then, from eqs. (13), (14) and (15), we obtain the analytic expression of the Young modulus of crystals with defects:

$$
\begin{aligned}
E= & E_{0}-\frac{n_{V} a}{v}\left\{\frac{a_{0}}{\theta}\left(\frac{\partial U_{0}}{\partial a}\right)^{2}\left(2+\frac{U_{0}}{2 \theta}\right)\right. \\
& \left.+\left(2 a_{0} \frac{\partial^{2} U_{0}}{\partial a^{2}}+\frac{\partial U_{0}}{\partial a}\right)\left(1+\frac{U_{0}}{2 \theta}\right)\right\},
\end{aligned}
$$

where $v=V / N$.

In the limit of small strain, the bulk modulus $K$ and shear modulus $G$ of crystals with defects are given by 

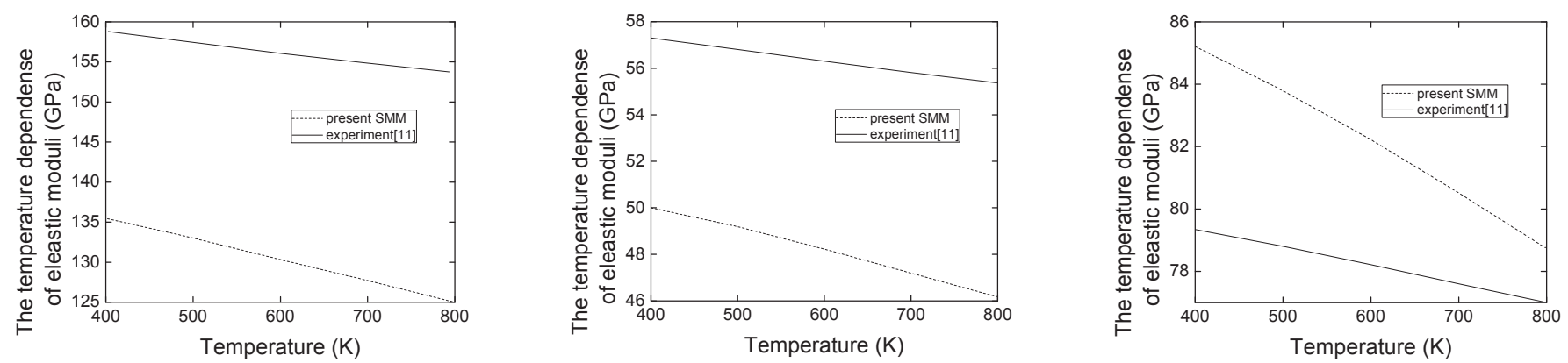

FIG. 1: Temperature dependence of elastic constants $C_{11}, C_{12}$ and $C_{44}$ of $\mathrm{Si}$ crystal with defects (equilibrium concentration of vacancies $n_{V} \sim 7.2 \times 10^{-13}$ at $\left.T=800 \mathrm{~K}\right)$.

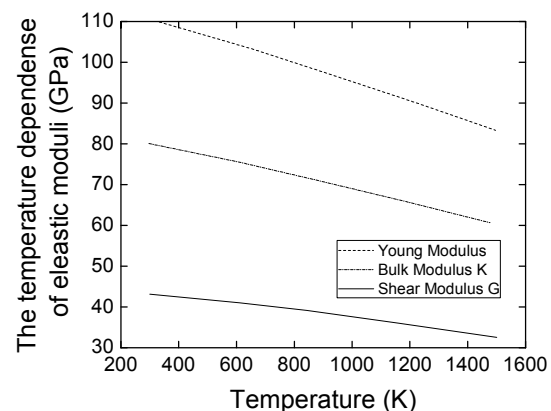

FIG. 2: Temperature dependence of elastic moduli $E, G$ and $K$ of $\mathrm{Si}$ crystal with defects. (equilibrium concentration of vacancies $n_{V} \sim 3.3 \times 10^{-7}$ at $\left.T=1500 \mathrm{~K}\right)$.

$$
\begin{aligned}
& K=\frac{E}{3(1-2 v)} \\
& G=\frac{E}{2(1+v)},
\end{aligned}
$$

where $\mathrm{v}$ denontes the Poisson's ratio, and the elastic constants $C_{i j}$ are determined as Refs. [8,9]:

$$
\begin{aligned}
C_{11} & =\frac{E(1-v)}{(1+v)(1-2 v)} \\
C_{12} & =\frac{E v}{(1+v)(1-2 v)} \\
C_{44} & =\frac{E}{(1+v)},
\end{aligned}
$$

[1] L. A. Girifalco, Statistical Physics of Materials (J. Wiley. Intersciens publ., Toronto, 1973) Mir 1975 (in Russian).

[2] V. I. Zubov, Phys. Status Solidi B 101, 95 (1980); ibid 113, K 73 (1982).

[3] V. V. Hung, N. T. Hai, and N. Q. Bau, J. Phys. Soc. Jpn. 66, 3494 (1997).

[4] N. Tang and V. V. Hung, Phys. Stat. Sol. B 149, 511 (1988); ibid B 161, 165 (1990).

\section{RESULTS AND DISCUSSION}

The elastic constants and moduli of the Si crystal with defects are calculated using the SMM calculation scheme [3-8] as well as using the many-body interaction potential [10].

In Fig.1, we present the elastic constants $C_{i j}$ of the $\mathrm{Si}$ crystal calculated by the SMM formalism, together with the experimental results [11]. Overall good agreements between the calculation and experimental results are obtained for a wide temperature range.

In Fig.2, we present the temperature dependence of the elastic moduli $E, G$ and $K$ of the Si crystal with defects as a function of the temperature $T$. The decreasing in the elastic constants $C_{i j}$ and moduli $E, G$ and $K$ indicates the stronger anharmonicity contribution of the thermal lattice vibration at high temperature.

\section{CONCLUSION}

In conclusion, we have presented the SMM formulation for the elastic moduli and constants of the diamond cubic semconductors with the point defects. The elastic moduli $E, G, K$ and constants $C_{i j}$ have been calculated successfully for the Si crystal with defects.

\section{Acknowledgments}

This work was supported by NAFOSTED (No.103 01 2609).

[5] V. V. Hung, H. V. Tich, and K. Masuda-Jindo, J. Phys. Soc. Jpn. 69, 2691 (2000).

[6] K. Masuda-Jindo, V. V. Hung, and P. D. Tam, Phys. Rev. B 67, 094301 (2003).

[7] K. Masuda-Jindo, S. R. Nishitani, and V. V. Hung, Phys. Rev. B 70, 184122 (2004).

[8] V. V. Hung, K. Masuda-Jindo, and N. T. Hoa, J. Mater Res. 22, 2230 (2007). 
[9] V. V. Hung, N. T. Hoa, Comm. in Phys. 15, 242 (2005).

[10] F. Stillinger and T. Weber, Phys. Rev. B 31, 5262 (1985).

[11] http://www.ioffe.ru/SVA/NSM/Semicond/ 